\title{
Fabrication and Characterization of Anisotropic Silk Fibroin/Gelatin Scaffolds Using Unidirectional Freezing
}

\author{
M.C.T. Asuncion ${ }^{1}$, C.H.J. Goh ${ }^{1,2}$, and S.L. Toh ${ }^{1,3}$ \\ ${ }^{1}$ Department of Biomedical Engineering, National University of Singapore \\ ${ }^{2}$ Department of Orthopaedic Surgery, National University of Singapore \\ ${ }^{3}$ Department of Mechanical Engineering, National University of Singapore
}

\begin{abstract}
Cells have been shown to respond to the topography of their environment through contact guidance, which can di-rect their migration, morphology and even gene expression. This is a particularly important phenomenon in tissue engineering, wherein the importance of recreating the native environment of cells is paramount to achieve a functional tissue-engineered con-struct which can readily integrate to the local environment upon implantation. In light of this, scaffolds with a certain alignment or anisotropy are being fabricated in order to recapitulate the natural anistropy in certain tissue such as muscles. However, most common fabrication methods are usually tedious and involve harsh conditions during processing. Unidirectional freezing is a simpler method which offers biocompatibility, versatility, tunability and scalability in
\end{abstract}

achieving anisotropic scaffolds. In this study, different silk fibroin/gelatin solutions were unidirectionally-frozen and subsequently lyophilized in order to create aniso-tropic scaffolds. Morphological, mechanical and cell proliferation properties were then investigated to fully characterize the scaf-folds and the effect of the addition of gelatin. Alignment was attained in these scaffolds, as well as anisotropy in terms of their mechanical properties. Furthermore, mesenchymal stem cells cultured on these scaffolds were shown to align themselves accord-ing to the direction of the fibers. Together, the results show a possibility of utilizing these scaffolds in engineering anisotropic tis-sues.

Keywords - silk, fibroin, gelatin, scaffold, anisotropic.

The original version of this chapter was inadvertently published with an incorrect chapter pagination 746 and DOI 10.1007/978-3-31932703-7_144. The page range and the DOI has been re-assigned. The correct page range is 752 and the DOI is 10.1007/978-3-31932703-7 145. The erratum to this chapter is available at DOI: 10.1007/978-3-319-32703-7_260

E. Kyriacou et al. (eds.), XIV Mediterranean Conference on Medical and Biological Engineering and Computing 2016,

IFMBE Proceedings 57,

DOI: $10.1007 / 978-3-319-32703-7 \_145$ 